\title{
A Trip-Chain Based User Equilibrium Traffic Assignment Model with Flexible Activities Scheduling Order
}

\author{
Chung-Yung Wang ${ }^{1}$ and Hsiang-Wu Chen ${ }^{2}$ \\ 1. Department of Logistics Management, Management College of National Defense University, Taipei 11258, Taiwan, R.O.C. \\ 2. Department of Transportation and Logistics Management, National Chiao Tung University, Hsinchu 30010, Taiwan, R.O.C.
}

\begin{abstract}
This study developed a user equilibrium traffic assignment model based on trip-chains with flexible activity scheduling order and derived the corresponding optimality conditions. We based on the gradient projection method to develop a solution algorithm, the accuracy of which was verified using the test network of UTown. This model could be used to estimate the transportation demands with and without activities scheduling restriction between OD (origin-destination) pairs based on trip-chains, as well as based on trips. Thus, the proposed model is more generalization than conventional trip based or trip-chain based traffic assignment models.
\end{abstract}

Key words: Traffic assignment, trip-chain, flexible activity scheduling order, gradient projection method.

\section{Introduction}

Traffic assignment is the core of the transportation planning. The transportation demands on each road of urban network can be estimated by the assumption of traveler route choice behavior through traffic assignment procedure. Conventional traffic assignment models use available information on road networks to distribute the trip demands between trip ends (OD (origin-destination) pairs) throughout a network. The supply and demand within a transportation system serve as a reference for the transportation planning. Analysis of traveler behavior usually includes multiple activities between OD pairs in a trip, which are presented using trip-chaining. In terms of home-work trips, the conventional trip based traffic assignment models consider only the route choices between the trip ends "home" and "work". However, the actual home-work trips often involve several secondary activities, such as dropping off children at school, making a deposit at the bank or arranging to send a package at the post office. These activities decompose

Corresponding author: Chung-Yung Wang, Ph.D., professor, research fields: transportation planning, traffic assignment problems, network design problem, trip-chain network equilibrium model and solution algorithm. a single home-work trip into a chain of secondary activities (home $\rightarrow$ school $\rightarrow$ bank $\rightarrow$ post office $\rightarrow$ work), thereby displaying the travel patterns associated with trip-chaining. Activities in the trip-chain and the order of activities influence travelers route choice but cannot be analyzed using conventional trip based traffic assignment models. That is, to develop a trip-chain user equilibrium traffic assignment model is an important research issue for transportation planning. This study is focused on model and solution algorithm development for trip-chain based traffic assignment model and organized as follows: The next section reviews literature relevant to rip-chain user route choice behaviors and models. The third section presents the development of a trip-chain based user equilibrium traffic model with flexible activity scheduling order. Then, we outline a gradient projection method used to develop a solution algorithm for the model and present an example of a derived solution and analysis of a road network in UTown. The final section presents conclusions of this study.

\section{Literature Review}

Hägerstrand [1] was the first researcher to discuss the trip-chain phenomenon and proposed the 


\section{A Trip-Chain Based User Equilibrium Traffic Assignment Model with Flexible Activities Scheduling Order}

activity-based travel behavior theory. Srinivasan [2] presented a definition of trip-chains as a series of activities scheduled in time and space, chaining together a work trip and one or more non-work trips. Primerano et al. [3] observed that the trip-chains of multiple activities can be divided into primary and secondary activities. The former refers to the primary purpose for which trips are initiated and which travel behavior between OD pairs is based on; The latter indicates activities that are performed during the completion of primary activities. In many empirical studies of traveler behaviors analysis from different social economic survey data also pointed out, such like Hensher and Reyes [4], McGuckin et al. [5], Morency and Valiquette [6], Currie and Delbosc [7] and Zhao et al. [8], the trip-chain phenomenon exists in the travel choice behavior of road users.

Based on the assumption that individuals display trip-chaining behavior, Lam and Yin [9] proposed a dynamic user equilibrium activity/route choices model. Through this model, activities participation can be considered in time-dependent route choice behaviors of travelers. Maruyama and Harata [10] argued that, if trip-chains were processed as single independent trips, the connections among trips could not be seen, which would lead to discrepancies in the forecasting of travel demands. To overcome this problem, they adopted the trip-chaining behavior of road users under the principle of static user equilibrium. Given an established order of activities and the presumption that they must be completed in the fixed activities sequence, they developed two-stage combined models of trip-chaining (including a combined trip distribution and traffic assignment model and a combined modal split and assignment model), solved using a traditional algorithm of linear approximation.

Maruyama and Harata [11] proposed a trip-chain based network equilibrium model. The model was grounded on the traffic assignment model with variable demand proposed by Beckmann et al. [12], which considered the trip-chaining patterns of road users in which the inverse demand function in the original objective function was modified as a function of trip-chain flow. A constraint of flow conservation in trip-chains was also added, thereby establishing the trip-chain network equilibrium model. Maruyama and Sumalee [13] proposed that a direct relationship exists between congestion pricing and trip-chaining behavior. They adopted the trip-chain based equilibrium model developed by Ref. [11] to discuss the effectiveness and fairness of cordon-based and area-based pricing schemes for congestion charges. In consideration of trip-chaining behavior, Higuchi et al. [14] proposed an innovative two-stage integration model, which included a network equilibrium model for the selection of transportation modes and routes. The integration model was built on variational inequality problems that were solved using the relaxation method. Kang et al. [15] developed an activity-based bilevel programming network design model. The bilevel formulation includes an upper level network design problem and a shortest path model as the lower level problem which is an activities scheduling optimization problem. The trip-chain based route choice behaviors of travelers have been involved in the lower level of network design model. But the user equilibrium route choice behaviors can not be restricted in their model.

From the reviews of above literatures, there are some directions to improve the trip-chaining assignment process. First direction is to allow the scheduling more realistic. The models of Refs. [10, 11] assume that the order in which activity locations in a trip-chain is given in advance and must be completed accordingly, that is, the scheduling of activities on a trip-chain is fixed in these models. About the studies of scheduling of activities on a trip-chain, Chung et al. [16] applied the dynamic SEM (structural equation model) to explore the relationship between activity participation and travel behavior. They suggest that the level-of-service variables, such like level of congestion in the transportation network, should be included in further extended models to measure the influence on the order 
of activity participation and travel behavior. Ettema et al. [17], Jenelius et al. [18] and Kang and Recker [19] all showed the scheduling flexibility of a trip chain and how to adjust the schedule of activities on a trip chain in response to travel time changes. Due to the sequence of different activities which will be derived different travel cost during a trip-chain, the activities scheduling becomes an important decision factor to influence the trip-chain route choice decision of road users. Secondly, to have a better computational algorithm, Maruyama and Harata [11] adopted a link-based approach similar to the Frank-Wolfe algorithm to solve their trip-chain traffic assignment model. The gradient projection method [20] is more efficient than Frank-Wolfe algorithm to solve the traffic assignment model. We could base on the structure of gradient projection method to develop a suitable solution algorithm.

\section{The Model}

This study take into consideration the trip-chaining characteristics. At first, we define the trip-chain based user equilibrium principle as follows.

No traveler can improve his/her trip-chain route travel time by unilaterally changing trip-chain route. That is, the route choice must pass all of the particular intermediary activity locations between their origin and destination and incur the minimal travel costs. Thus, if trip-chain route between OD pair $(r, s)$ is being used, the trip-chain route travels costs will equal the minimal trip-chain route travel cost between OD pair $(r, s)$. As for the other unused trip-chain routes, the travel costs must be equal to or greater than the minimal trip-chain route travel cost between the OD pair $(r, s)$.

\subsection{The Definitional Constraint of Trip-Chain Routes}

Before we formulate the trip-chain based user equilibrium traffic assignment model, we should consider how to express the route flow between the trip ends which passing the locations of all the trip-chain activities. We define $N^{r s}$ is the set of activity locations (nodes) that the trip-chain route must pass between OD pair $(r, s)$. Here, an activity node/path indicator variable $\bar{\gamma}_{n p}^{r s}$ is designed, where $n \in N^{r s}$. The variable $\bar{\gamma}_{n p}^{r s}$ is a zero-one variable. When route $p$ between OD pair $(r, s)$ passes the activity node $n$ on the trip-chain, then $\bar{\gamma}_{n p}^{r s}=1$; otherwise, $\bar{\gamma}_{n p}^{r s}=0$. The flow on trip-chain routes between OD pair $(r, s)$ must pass all of the activity nodes on the trip-chain between the given OD pair and can be expressed as Eqs. (1) and (2):

$$
\begin{gathered}
h_{\hat{p}}^{r s}=h_{p}^{r s} \prod_{n \in N^{r s}} \bar{\gamma}_{n p}^{r s}, \forall r, s, p \in(r, s), \hat{p} \in(r, s) \\
\bar{\gamma}_{n p}^{r s} \in\{0,1\}, \forall r, s, n \in N^{r s}, p \in(r, s)
\end{gathered}
$$

Eq. (1) is the definitional constraint of trip-chain routes, representing the relationship between $h_{\hat{p}}^{r s}$, the flow of trip-chain routes that pass the locations of trip-chain activities between OD pair $(r, s)$, and $h_{p}^{r s}$, the flow of general routes between OD pair $(r, s)$. In Eq. (1), $\bar{\gamma}_{n p}^{r s}$ is a $\{0,1\}$ indicator variable, and $N^{r s}$ is the set of activity locations (or nodes) $n$ that the trip-chain route must pass between OD pair $(r, s)$, $n \in N^{r}$. With consideration to flexible activities scheduling order, there is no set order to activities $n_{1}, n_{2}, \ldots, n_{i}$. In contrast, if the order of the activity set is fixed in the sequence of $\left\{n_{1}, n_{2}, \ldots, n_{i}\right\}$, the activities scheduling fixed restriction will be enabled. If route $p$ between OD pair $(r, s)$ passes all of the activity nodes on the trip-chain between the OD pair, then multiply all indicator variables $\bar{\gamma}_{n p}^{r s}$ will be equal to 1 , and it can be expressed as $\prod_{n \in N^{r s}} \bar{\gamma}_{n p}^{r s}=1$. We can obtain $h_{\hat{p}}^{r s}=h_{p}^{r s} \prod_{n \in N^{r s}} \bar{\gamma}_{n p}^{r s}=h_{p}^{r s} ;$ If one or more of activity nodes on the trip-chain were not passed, then $\prod_{n \in N^{r s}} \bar{\gamma}_{n p}^{r s}=0$, and $h_{\hat{p}}^{r s}=h_{p}^{r s} \prod_{n \in N^{r s}} \bar{\gamma}_{n p}^{r s}=0$. Therefore, Eq. (1) shows that the flow of trip-chain routes between OD pair $(r, s)$ must pass all of the activity nodes on the trip-chain between the given OD pair. The activity node/path variable $\bar{\gamma}_{n p}^{r s}$ is a zero-one variable as constrained in Eq. (2). 


\subsection{Model Formulation}

To consider the characteristic of trip-chain based user equilibrium traffic assignment problem, the OD pair between a trip-chain should be located between two different nodes. Trip-chains are assigned the following generalized definition: a series of activities (secondary activities) between the origin and destination (primary activity). When travelers engage in trip-chaining, besides the origin and destination nodes, they also must complete (pass) all of the activities (location nodes). Under the assumption of flexible activity scheduling order, the scheduling of activities of a trip-chain also should be concerned in the trip-chain based traffic assignment model. The order of activities makes no difference to the travelers' trip purpose but affect to the total trip cost due to the different route choice behavior.

The proposed model is formulated as follows:

$$
\min z(\mathbf{x})=\sum_{a} \int_{0}^{x_{a}} c_{a}(\omega) \mathrm{d} \omega
$$

Subject to constraint of flow conservation in trip-chain:

$$
\sum_{\hat{p}} h_{\hat{p}}^{r s}=\bar{q}^{r s}, \forall r, s, \hat{p}
$$

Non-negative constraint of flow in trip-chain:

$$
h_{\hat{p}}^{r s} \geq 0, \forall r, s, \hat{p}
$$

Definitional constraint:

$$
\begin{gathered}
x_{a}=\sum_{r} \sum_{s} \sum_{\hat{p}} h_{\hat{p}}^{r s} \bar{\delta}_{a \hat{p}}^{r s} \geq 0, \forall a \\
\bar{\delta}_{a \hat{p}}^{r s}=\{0,1\}, \forall r, s, a, \hat{p}
\end{gathered}
$$

Definitional constraint of trip-chain routes:

$$
\begin{gathered}
h_{\hat{p}}^{r s}=h_{p}^{r s} \prod_{n \in N^{r s}} \bar{\gamma}_{n p}^{r s}, \forall r, s, p \in(r, s), \hat{p} \in(r, s) \\
\bar{\gamma}_{n p}^{r s}=\{0,1\}, \forall r, s, n \in N^{r s}, p
\end{gathered}
$$

where, Eq. (3) is an objective function of user equilibrium. Eq. (4) presents the constraint of flow conservation in the trip-chains, indicating that the total flow on the trip-chain routes between any given trip-chain OD pair of $(r, s)$ with corresponding trip-chain activity nodes must equal $\bar{q}^{r s}$, the trip-chain demand of the given trip-chain OD pair. Eq. (5) is the non-negative constraint of trip-chain flow, and Eq. (6) presents the definitional constraint, which indicates the relationship between the traffic flow on each link in the network and the flow on the routes in the trip-chain; In Eq. (7), $\bar{\delta}_{a \hat{p}}^{r s}$ is a $\{0,1\}$ indicator variable that equals 1 when trip-chain route $\hat{p}$ passes link $a$ and 0 when it does not. Eqs. (8) and (9) are the definitional constraint of trip-chain routes. There is no set sequence to activities $n_{1}, n_{2}, \ldots, n_{m}$.

In solving the above optimization problem, one can incorporate Lagrangean multipliers $\hat{\pi}^{r s}$ into the objective function, and obtain the following Lagrangean function:

$$
\begin{aligned}
& \min L(z(\mathbf{x}), \pi) \\
& =\sum_{a} \int_{0}^{x_{a}} c_{a}(\omega) \mathrm{d} \omega+\hat{\pi}^{r s}\left(\bar{q}^{r s}-\sum_{\hat{p}} h_{\hat{p}}^{r s}\right)
\end{aligned}
$$

Subject to:

$$
h_{\hat{p}}^{r s} \geq 0, \forall r, s, \hat{p}
$$

We then calculated the first-order partial derivatives of the decision variable $h_{\hat{p}}^{r s}$ and the Lagrange multiplier $\hat{\pi}^{r s}$ in the Lagrange function of Eq. (10) to obtain the optimization conditions, Eqs. (12)-(14):

$$
\begin{gathered}
h_{\hat{p}}^{r s}\left(c_{\hat{p}}^{r s}-\hat{\pi}^{r s}\right)=0 \\
h_{\hat{p}}^{r s}=h_{p}^{r s} \prod_{n \in N^{r s}} \bar{\gamma}_{n p}^{r s} \geq 0 \\
\left(c_{\hat{p}}^{r s}-\hat{\pi}^{r s}\right) \geq 0
\end{gathered}
$$

Eqs. (12)-(14) represent the complementary slackness relationships in trip-chain route choice behaviors for road users, which means that the travel times $c_{\hat{p}}^{r s}$ of the trip-chain path $\hat{p}$ being assigned traffic flows $h_{\hat{p}}^{r s}$ for a given OD pair $(r, s)$ are equal to the minimum path travel times $\hat{\pi}^{r s}$, otherwise trip-chain path travel times $c_{\hat{p}}^{r s}$ between each OD $(r, s)$ pair are greater than or equal to the minimum trip-chain path travel times $\hat{\pi}^{r s}$. The optimality 
condition of the proposed model is consistent with the trip-chain based user equilibrium principle.

\section{The Solution Algorithm}

In this study, the GP (gradient projection) method is used to develop the corresponding solution algorithm. The steps are explained in detail below:

- Step 0: initialization of algorithm;

- Step 0.1: Let $n=0$, set the free flow travel time $\left\{c_{a_{0}}\right\}$ as the travel time of the initial solutions for each link in the network and calculate the shortest route passing all activity nodes between the trip-chain OD pair $(r, s)$;

- Step 0.2: Based on the initial solution, create a set of trip-chain routes and define $h_{\hat{p}}^{r s}=\bar{q}^{r s}, \forall r, s$, the trip-chain traffic flow passing each activity node between trip-chain OD pair $(r, s)$, as $\left\{h_{\hat{p}}^{r s}\right\}^{(n+1)}$;

- Step 1: computing operations for master problem;

- Step 1.1: Let $n=n+1$, calculate traffic flow on each link based on $\left\{h_{\hat{p}}^{r s}\right\}^{(n)}$, the trip-chain path flow between trip-chain OD pair $(r, s)$. Then renew the travel time for each link in the network to $\left\{c_{a}^{(n)}(\mathrm{x})\right\}$;

- Step 1.2: Calculate the shortest paths that pass the trip-chain activity nodes between each trip-chain OD pair $(r, s)$ and place them in the first route $\hat{p}^{r s^{*}}$ of feasible trip-chain route $\operatorname{set}\left\{P_{\hat{p}}^{r s}\right\}^{(n)}$;

- Step 2: Computing operations for restricted master problem;

Step 2.1: Use Eq. (15) to renew trip-chain path flow $\left\{h_{\hat{p}}^{r s}\right\}^{(n+1)}$ :

$$
h_{\hat{p}}^{r s}{ }^{(n+1)}=\max \left\{0,\left(h_{\hat{p}}^{r s}{ }^{(n)}+\alpha_{\hat{p}}^{r r^{(n)}} d_{\hat{p}}^{r s}(n)\right)\right\}
$$

Let the shortest route that passes the activity nodes on the trip-chain between OD pair $(r, s)$ in the route set be $\hat{p}^{*}$. Using Eq. (16), we can derive the shortest trip-chain path flow as follows:

$$
h_{\hat{p}^{*}}^{r s}=\bar{q}^{r s}-\sum_{\hat{p} \neq \hat{p}^{*}} h_{\hat{p}}^{r s}
$$

The link flow $x_{a}^{(n+1)}$ can be derived as follows:

$$
x_{a}^{(n+1)}=\sum_{r s} \sum_{\hat{p}} h_{\hat{p}}^{r r^{(n+1)}} \bar{\delta}_{a \hat{p}}^{r s}, \quad \forall a
$$

- Step 2.2: Perform convergence testing. If the link flow values of two consecutive rounds differ by less than convergence criterion $\varepsilon$, as shown in Eq. (18), then convergence is achieved. Otherwise, return to Step 1:

$$
\max _{a}\left|\frac{x_{a}^{(n+1)}-x_{a}^{(n)}}{x_{a}^{(n)}}\right| \leq \varepsilon
$$

The descent direction $d^{(n)}$ in Step 2.1 is the first order derivative of the trip-chain route variable $h_{\hat{p}}^{r s}$ in objective Eq. (3), as shown below:

$$
\frac{\partial z(\mathbf{x})}{\partial h_{\hat{p}}^{r s}}=c_{\hat{p}}^{r s}-c_{\hat{p}^{*}}^{r s}, \forall r \in R, s \in S, \hat{p} \neq \overline{\hat{p}}
$$

Step size $\alpha^{(n)}$ in Step 2.1 is the reciprocal of the second order derivative of the trip-chain route variable $h_{\hat{p}}^{r s}$ in objective Eq. (3):

$$
\frac{\partial^{2} z(\mathbf{x})}{\partial\left(h_{\hat{p}}^{r s}\right)^{2}}=\sum_{a} c_{a}^{\prime} \bar{\delta}_{a \hat{p}}^{r s}+\sum_{a} c_{a}^{\prime} \bar{\delta}_{a \hat{p}^{*}}^{r s}-\sum_{a \in\left\{\hat{p} \cap \hat{p}^{*}\right\}} 2 c_{a}^{\prime}
$$

Thus:

$$
\alpha_{p}^{r s}=\frac{v}{\sum_{a} c_{a}^{\prime} \bar{\delta}_{a \hat{p}}^{r s}+\sum_{a} c_{a}^{\prime} \bar{\delta}_{a \hat{p}{ }^{*}}^{r s}-\sum_{a \in\left\{\hat{p} \cap \hat{p}^{*}\right\}} 2 c^{\prime}{ }^{\prime}}
$$

where, parameter $v$ is a constant range between 0 and 1 ; A value closer to 1 indicates a faster solution process whereas a value closer to 0 indicates higher precision.

When computing the master problem in the above solution algorithm, the process of solving the shortest trip-chain route solution is as follows:

- Step 0: Set the origin, destination and intermediary activity nodes in the trip-chain;

- Step 1: Use the shortest route algorithm to derive the shortest routes and distances between the origin and all of the activity nodes, between activity nodes and the other activity nodes, and between all of the activity nodes and the destination;

- Step 2: Use forward dynamic programming or backward dynamic programming to establish all of the route combinations that pass the nodes of the secondary activities between the origin and the destination;

- Step 3: Substitute the shortest route combination 
obtained in Step 2 into the original network to derive the shortest trip-chain route, which is the shortest route solution that passes the nodes all of the trip-chain activities.

\section{Numerical Example}

To understand the characteristics of the proposed trip-chain based equilibrium traffic assignment model and verify the accuracy of the derived solutions, we performed a test based on the UTown test network (Fig. 1) [21]. Trip demand data were drawn up for nine trip-chains between five nodes serving as origins and destinations. For trip-chain Nos. 1, 4 and 8, the trip-chain OD pairs were 1 5, 3 5 and 5 1, with no secondary activities in between. Trip-chain Nos. 2, 3, 5, 6,7 and 9 were required to pass the nodes of the secondary activities between the trip-chain OD pairs. The details and trip demands of each trip-chain OD pair are displayed in Table 1. The road network of UTown comprised 42 nodes and 124 links. The relevant network data, including link numbers, links, free flow travel costs, and link capacity that we can cite from Ref. [21]. Here, we assume that the travel cost function of each link was adopted by FHWA (U.S. Federal Highway Administration) cost function, as shown in Eq. (22):

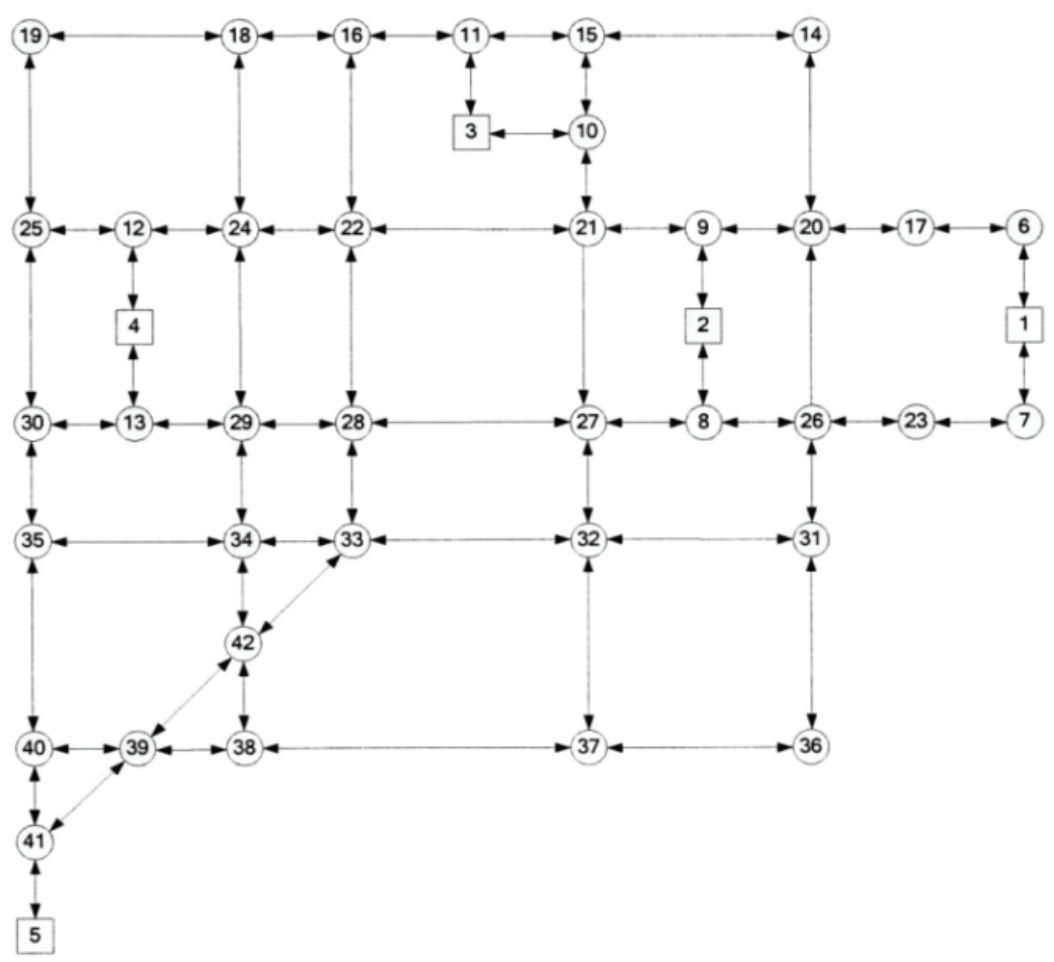

Fig. 1 The UTown test network.

Table 1 Activity nodes on the trip-chains and trip demands between trip-chain OD pairs.

\begin{tabular}{llllc}
\hline Trip-chain number & Origin & Activity node & Destination & Trip demands \\
\hline 1 & 1 & - & 5 & 2,050 \\
2 & 1 & 2,3 & 5 & 1,880 \\
3 & 2 & 3 & 4 & 850 \\
4 & 3 & - & 5 & 1,080 \\
5 & 3 & 2,4 & 5 & 850 \\
6 & 4 & 2,3 & 1 & 850 \\
7 & 5 & $2,3,4$ & 1 & 850 \\
8 & 5 & - & 1 & 2,860 \\
9 & 5 & 3 & 1 & 540 \\
\hline
\end{tabular}




$$
c_{a}\left(x_{a}\right)=c_{a_{0}}\left(1+0.15\left(\frac{x_{a}}{C a p_{a}}\right)^{4}\right), \forall a \in A
$$

Based on network and trip-chain OD pair data, we adopted the solution algorithm developed in Section 4. A program was written using the Borland $\mathrm{C}++$ editor and testing was performed on a personal computer with an Intel Pentium $43.4 \mathrm{~GB} \mathrm{~Hz}$ processor. Testing demonstrated that the results of the proposed model could be determined, such as the path flows and travel costs of the trip-chains in Appendix A. Based on the results, we performed the following analysis:

(1) The tables contain three trip-OD pairs without trip-chain activities (Nos. 1, 4 and 8) and six trip-chain OD pairs with trip-chain activities (Nos. 2, $3,5,6,7$ and 9);

(2) Regardless of whether activity nodes exist on the trip-chains between trip-chain OD pairs, the travel times of the same trip-chain route between trip-chain OD pairs remained the same. Furthermore, in the trip-chain OD pairs with trip-chain activity nodes, all the set activity nodes have been passed, thereby satisfying the optimization conditions of Eqs. (12)-(14). This also follows the trip-chain based user equilibrium principle, thereby demonstrating the accuracy of the obtained results. The computation time was $2 \mathrm{~s}$, which was deemed acceptable;

(3) Trip-chains Nos. 1 and 2 illustrate how the route selection results and the links may differ among different trip-chains between the same OD pair. Trip-chains Nos. 4 and 5 and Nos. 7, 8 and 9 also demonstrate this phenomenon. This shows that in the analysis of route choice behavior displayed by travelers, major errors are likely to occur between actual route choice behavior and the travel demands forecasted by conventional trip based traffic assignment models, which tend to neglect the activity demands of road users in trip-chains. This also explains the importance of developing traffic assignment models based on trip-chains;

(4) Considering the activities nodes passed schedule flexible in the model, we can also find that between the trip-chain OD pair $(1,5)$, a number of routes may first pass Activity Node 2 before passing Activity Node 3, whereas others routes may first pass Activity Node 3 before passing Activity Node 2. This is consistent with our definition of trip-chains, where the order in which the necessary activities (nodes) other than the origin and destination are completed (passed) makes no difference, but is determined by the costs that travelers consider in the selection of routes;

(5) The route choice results show that in order to pass every activity node in the trip-chain, the nodes of some trip-chain links may be passed twice. For example, in the first route for trip-chain No. 2 in Appendix A, a portion of the route is $21 \rightarrow 10 \rightarrow 3 \rightarrow 10 \rightarrow 21$; Both Nodes 21 and 10 were passed twice, but the activity nodes that had to be passed on the trip-chain route were passed no more than once.

\section{Conclusions}

This study presents the following conclusions and contributions:

(1) We designed a trip-chain route definition constraint. Through the activity node/path indicator variable $\bar{\gamma}_{n p}^{r s}$, the flows relationship between common route and trip-chain route between each trip-chain OD pair and can be expressed easily. Whether the phenomenon of activities scheduling is fixed or not, all can be expressed by the trip-chain route definition constraint;

(2) We developed a user equilibrium traffic assignment optimization model based on trip-chains with flexible activity scheduling order, the results of which are consistent with the principle of trip-chain based user equilibrium. In the solution, we based on the gradient projection method to develop a solution algorithm, the accuracy of which was verified using the test network of UTown. The model developed in this study could be used to calculate the trip demands with and without activities scheduling restriction between OD pairs based on trips as well as based on 
trip-chains. Therefore, the developed model is more generalizable than conventional trip based or trip-chain based traffic assignment models;

(3) When collecting information related to the activity nodes on trip-chains of road users for future transportation planning, applying the proposed model could facilitate a more reasonable estimation of demand and supply relationships in urban traffic. In addition, the investment of transportation resources by traffic management departments could better satisfy the actual demands of travelers.

\section{Acknowledgments}

We sincerely thank the Ministry of Science and Technology of Taiwan (Project No: MOST 103-2410-H-606 -006) for funding this study.

\section{References}

[1] Hägerstrand, T. 1970. "What about People in Regional Science." Papers of The Regional Science Association 24 (1): 7-21.

[2] Srinivasan, S. 1998. "Linking Land Use, Transportation and Travel Behavior: Understanding Trip Chaining in Terms of Land Use and Accessibility Patterns, Cambridge." M.Sc. thesis, Department of Urban Studies and Planning, Massachusetts Institute of Technology.

[3] Primerano, F., Taylor, M., Pitaksringkarn, L., and Tisato, P. 2008. "Defining and Understanding Trip Chaining Behaviour." Transportation 35: 55-72.

[4] Hensher, D., and Reyes, A. 2000. "Trip Chaining as a Barrier to the Propensity to Use Public Transport." Transportation 27: 341-61.

[5] McGuckin, N., Zmud, J., and Nakamoto, Y. 2005. "Trip Chaining Trends in the United States: Understanding Travel Behavior for Policy Making." Transportation Research Record 1917: 199-204.

[6] Morency, C., and Valiquette, F. 2010. "Trip Chaining and Its Impact on Travel Behaviour. Activity-Based Analysis and Modeling." Presented at 12th World Conference on Transport Research, Lisbon, Portugal.

[7] Currie, G., and Delbosc, A. 2011. "Exploring the Trip Chaining Behaviour of Public Transport Users in Melbourne.” Transport Policy 18: 204-10.

[8] Zhao, Z., Chua, G., and Zhao, J. 2012. "Evolution of Trip Chaining Patterns in London from 1991 to 2010.
Innovations in Improving the Sensitivity of Models." Presented at 4th Conference on Innovations in Travel Modeling, Tampa, USA.

[9] Lam, W. H. K., and Yin, Y. 2001. "An Activity-Based Time-Dependent Traffic Assignment Model." Transportation Research Part B 35 (6): 549-74.

[10] Maruyama, T., and Harata, N. 2005. "Incorporating Trip Chaining Behavior in Network Equilibrium Analysis." Transportation Research Record 1921: 11-8.

[11] Maruyama, T., and Harata, N. 2006. "Difference between Area-Based and Cordon-Based Congestion Pricing: Investigation by Trip-Chain-Based Network Equilibrium Model with Non-additive Path Costs." Transportation Research Record 1964: 1-8.

[12] Beckmann, M. J., McGuire, C. B., and Winsten, C. B. 1956. Studies in the Economics of Transportation. New Haven: Yale University Press.

[13] Maruyama, T., and Sumalee, A. 2007. "Efficiency and Equity Comparison of Cordon- and Area-Based Road Pricing Schemes Using a Trip-Chain Equilibrium Model." Transportation Research Part A 41 (7): 655-71.

[14] Higuchi, T., Shimamoto, H., Uno, N., and Shiomi, Y. 2001. "A Trip-Chain Based Combined Mode and Route Choice Network Equilibrium Model Considering Common Lines Problem in Transit Assignment Model." Procedia Social and Behavior Sciences 20: 354-63.

[15] Kang, J. E., Chow, J. Y. J., and Recker, W. W. 2013. "On Activity-Based Network Design Problems." Transportation Research Part B 57: 398-418.

[16] Chung, J. H., Kim, T., Baik, H., and Choi, Y. S. 1995. “A Structural Equation Model of Activity Participation and Travel Behavior Using Longitudinal Data." Transportation Planning and Technology 32 (2): 163-85.

[17] Ettema, D., Bastin, F., Polak, J., and Ashiru, O. 2007. "Modelling the Joint Choice of Activity Timing and Duration." Transportation Research Part A 41 (9): $827-41$.

[18] Jenelius, E., Mattsson, L. G., and Levinson, D. 2011. "Traveler Delay Costs and Value of Time with Trip Chains, Flexable Activity Scheduling and Information." Transportation Research Part B 45 (5): 789-807.

[19] Kang, J. E., and Recker, W. 2013. "The Location Selection Problem for the Household Activity Pattern Problem." Transportation Research Part B 55: 75-97.

[20] Jayakrishnan, R., Tsai, W. K., Prashker, J. N., and Rajadhyaksha, S. 1994. "A Faster Path-Based Algorithm for Traffic Assignment." Transportation Research Record 1443: 75-83.

[21] U.S. Department of Transportation. 1986. UTPS Utown Case Study. Washington, D.C.: U.S. Department of Transportation. 


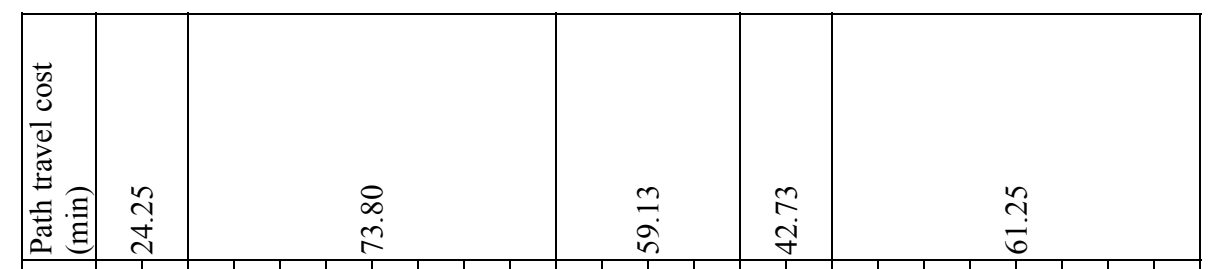

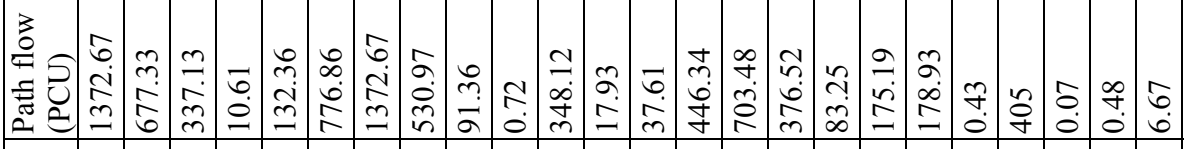

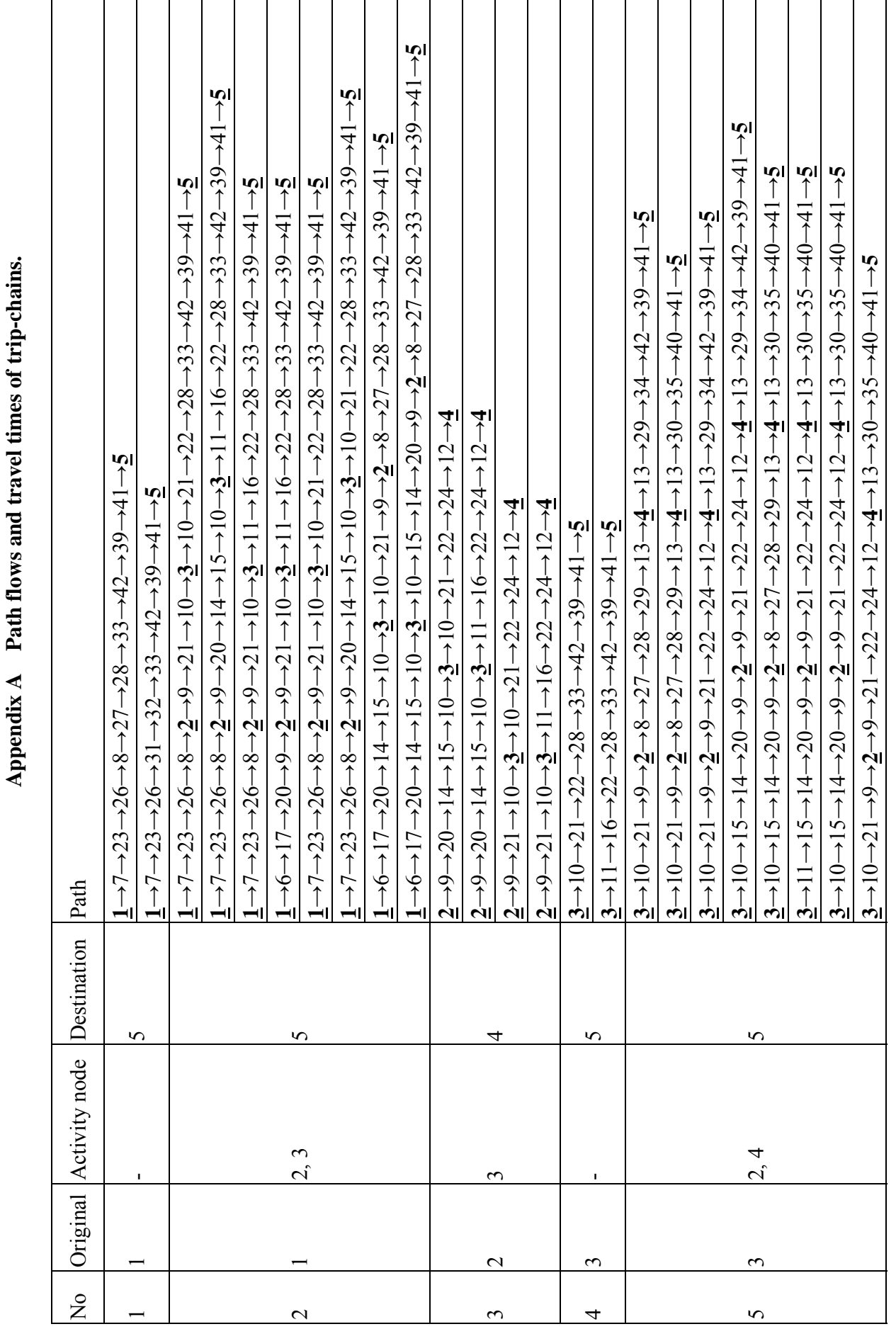




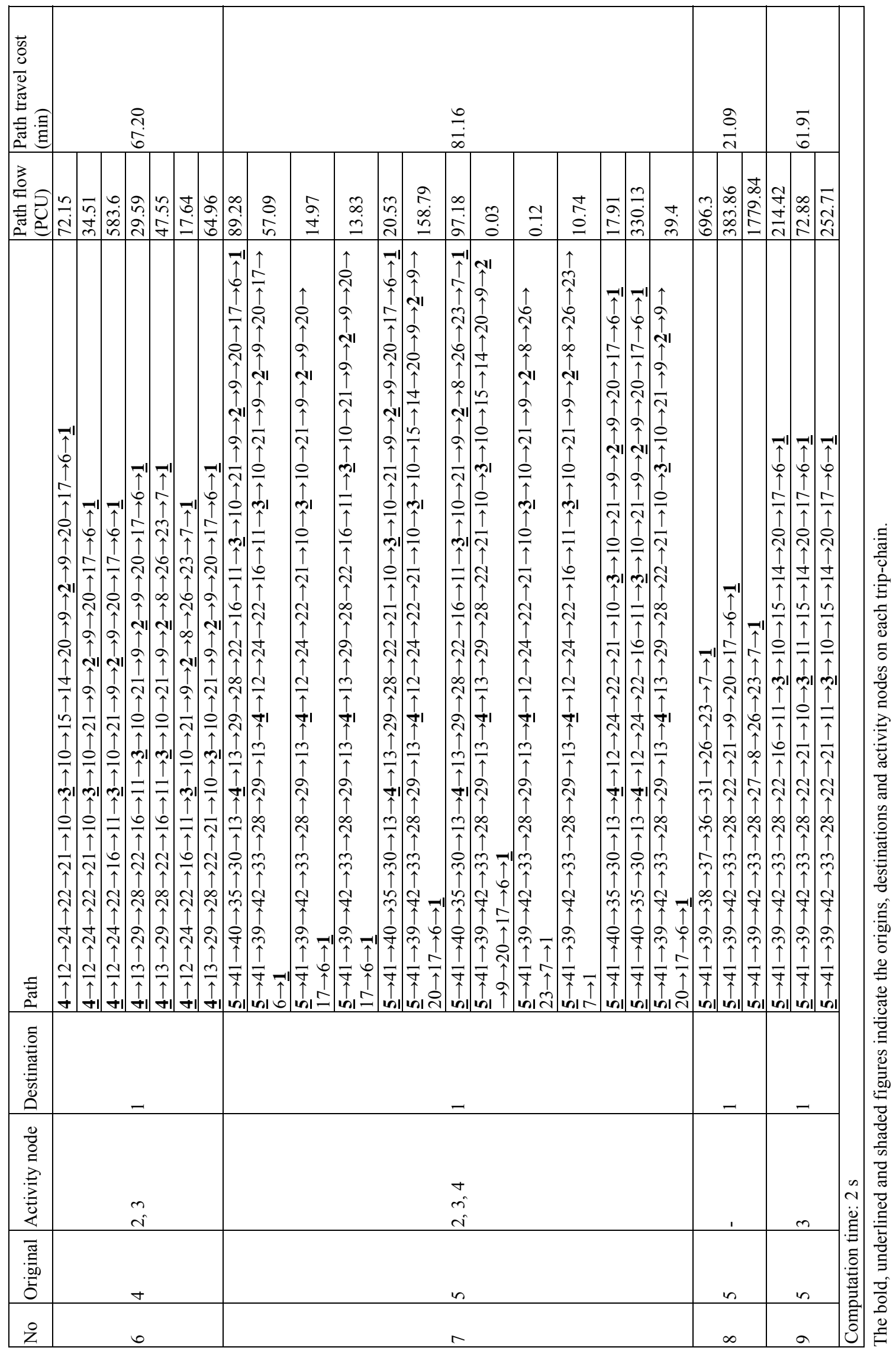

